\title{
UNIFORMIDADE DE APLICAÇÃO DE ÁGUA DE EQUIPAMENTOS AUTOPROPELIDOS DE IRRIGAÇÃO COM ASPERSORES DE PERFIL RADIAL TRIANGULAR, ELÍPTICO E RETANGULAR
}

\author{
GIULIANI DO PRADO ${ }^{1}$, ALBERTO COLOMBO², HENRIQUE F. E. DE OLIVEIRA ${ }^{3}$, \\ LESSANDRO C. FARIA ${ }^{4}$
}

\begin{abstract}
RESUMO: Este trabalho apresenta resultados de simulações digitais da uniformidade de aplicação de água de sistemas autopropelidos de irrigação, para a condição de ausência de ventos. Nas simulações foram considerados aspersores com perfis radiais de distribuição de água tipo triangular, elíptico e retangular, operando sob diferentes combinações de ângulo de giro (de 180 até $360^{\circ}$ ) e espaçamento entre carreadores (de 0 a $100 \%$ do diâmetro molhado - DM). Os resultados das simulações foram apresentados em três grupos distintos, cada um representando a mesma forma geométrica do perfil radial de distribuição de água. Independentemente do ângulo de giro, os melhores valores de uniformidade foram obtidos com espaçamentos menores que $45 \%$ e entre $55 \%$ e $70 \%$ do DM para o perfil radial triangular e com espaçamentos menores que $50 \%$ e entre $70 \%$ e 90\% do DM para os perfis radiais elíptico e retangular. Para os três perfis radiais, ângulos de giro do aspersor entre 180 e $230^{\circ}$ geram maiores valores de coeficiente de uniformidade.
\end{abstract}

PALAVRAS-CHAVE: simulações digitais, ângulo de giro, espaçamento entre carreadores.

\section{WATER APPLICATION UNIFORMITY OF SELF-PROPELLED IRRIGATION EQUIPMENT WITH SPRINKLERS PRESENTING TRIANGULAR, ELLIPTICAL AND RECTANGULAR RADIAL WATER DISTRIBUTION PROFILES}

\begin{abstract}
This study presents digital simulation results of water application uniformity of selfpropelled irrigation systems, under windless conditions. In the digital simulations, we considered sprinklers with triangular, elliptical and rectangular radial water distribution profiles, working under different combinations of angle of rotation (from $180^{\circ}$ to $360^{\circ}$ ) and spacing between carriers (from 0 to $100 \%$ of the wet diameter - WD). Simulation results were presented in three distinct groups, each one representing the same geometric shape of the radial water distribution profile. Regardless of the angle of rotation, the best values of uniformity were obtained with spacing lower than $45 \%$ and between $55 \%$ and $70 \%$ of WD for the triangular radial profile, and with spacing lower than $50 \%$ and between $70 \%$ and $90 \%$ of WD for the elliptical and rectangular radial profile. For the three radial distribution profiles, the sprinkler angles of rotation which range between $180^{\circ}$ and $230^{\circ}$ generate higher values of uniformity coefficient.
\end{abstract}

KEYWORDS: digital simulations, angle of rotation, spacing between carriers.

\footnotetext{
${ }^{1}$ Doutor, Prof. Adjunto, Departamento de Engenharia Agrícola - DEA, Universidade Estadual de Maringá - UEM, Cidade Gaúcha PR, Fone: (0xx44) 3675-1779, gprado@uem.br.

${ }^{2}$ Doutor, Professor Adjunto, Departamento de Engenharia - DEG, Universidade Federal de Lavras - UFLA, Caixa postal 37, Lavras MG, Fone: (0xx35) 38291388, acolombo@ufla.br.

${ }^{3}$ Eng ${ }^{\mathrm{o}}$ Agrícola, Doutorando, Departamento de Engenharia - DEG, Universidade Federal de Lavras - UFLA, Caixa postal 37, Lavras - MG, Fone: (0xx35) 3829-1388, henrique.ellias@ hotmail.com.

${ }^{4}$ Doutor, Prof. Adjunto, Centro de Desenvolvimento Tecnológico - CDTec, Engenharia Hídrica, Universidade Federal de Pelotas UFPel, Pelotas - RS, Fone: (0xx53) 3921-1240, lessandro.faria@ufpel.edu.br.

Recebido pelo Conselho Editorial em: 15-10-2009

Aprovado pelo Conselho Editorial em: 7-2-2012
} 


\section{INTRODUÇÃO}

A irrigação por aspersão apresenta-se como um dos métodos de irrigação mais empregados no Brasil. A grande aceitação desse método de irrigação deve-se, principalmente, à facilidade operacional que os diferentes sistemas por aspersão proporcionam. Inserida nesse contexto, a irrigação por aspersão com sistemas autopropelidos, devido à expansão do setor sucroalcooleiro (SATURNINO, 2006), vem sendo muito empregada na irrigação da cana-de-açúcar.

Na elaboração de um projeto e na operação de um sistema de irrigação, um dos fatores que devem ser levados em consideração é a distribuição de água do equipamento. Segundo PAZ et al. (2002) e ÁLVAREZ et al. (2004), sistemas de irrigação que não apresentam uma adequada distribuição de água não são apropriados quando o objetivo é o máximo retorno econômico. Conforme esses autores, a irrigação com maior uniformidade de aplicação de água proporciona melhores resultados econômicos em qualquer combinação de preço do produto e custo da água.

Nos sistemas autopropelidos de irrigação, vários fatores devem ser considerados para se alcançar adequada uniformidade de aplicação de água. Dentre esses fatores, têm-se aqueles influenciados pelo operador, tais como: i) ângulo de lançamento do jato de água do aspersor; ii) tipo de bocal do aspersor; iii) pressão de serviço do aspersor; iv) ângulo de giro do aspersor; v) características do perfil estacionário de distribuição de água do aspersor; vi) sobreposição dos perfis radiais, e vii) variações na velocidade de deslocamento do aspersor ao longo do carreador (PRADO et al., 2007; COLLIER \& ROCHESTER, 1980; COLOMBO, 1991) e aqueles influenciados pelas condições climáticas, tais como: i) velocidade do vento (FARIA et al., 2009), e ii) direção do vento (PRADO, 2008; SMITH et al., 2008; SHULL \& DYLLA, 1976).

Em função das inúmeras combinações operacionais possíveis, nas quais um sistema autopropelido de irrigação pode trabalhar, é extremamente difícil ou mesmo impraticável determinar, em ensaios de campo, como os realizados por ROCHA et al. (2005), as combinações operacionais que proporcionam melhor uniformidade de aplicação de água. Desta forma, aplicativos computacionais são importante ferramenta para simular a distribuição espacial da água aplicada. Estes podem considerar as inúmeras condições operacionais nas quais o sistema pode trabalhar e encontrar as combinações operacionais que levam a adequadas condições de uniformidade de aplicação de água (PRADO \& COLOMBO, 2006).

Para elaboração destas simulações, em aplicativos computacionais, a exemplo do SIMUASOFT (PRADO, 2004) e TRAVGUN (NEWELL et al., 2003), é necessário o conhecimento do perfil radial de distribuição de água dos aspersores. Segundo PRADO \& COLOMBO (2005), pela dificuldade de levantar essas informações para canhões hidráulicos, os fabricantes nem sempre disponibilizam esses dados técnicos. Desta forma, devido à restrita gama de informações disponíveis, é comum a utilização de perfis radiais de distribuição de água com formato geométrico típico.

A utilização de perfis típicos em simulações digitais pode ser empregada, pois muitos canhões hidráulicos, operando em adequadas condições operacionais de pressão de serviço e diâmetros de bocais, assumem uma forma geométrica que tende aos perfis $\mathrm{C}$ (elíptico) e $\mathrm{F}$ (retangular) apresentadas por CHRISTIANSEN (1942), como nos trabalhos desenvolvidos por: PRADO \& COLOMBO (2007) e CÉSAR et al. (2004). Também ROLIM \& PEREIRA (2005), na simulação da distribuição espacial da água aplicada por sistemas autopropelidos, consideram apenas o perfil radial com formato geométrico A (triangular) descrito por CHRISTIANSEN (1942).

No trabalho pioneiro de CHRISTIANSEN (1942), foram analisados os efeitos de seis formas geométricas distintas (A, B, C, D, E e F) do perfil de distribuição sobre os critérios de espaçamento entre aspersores, em sistemas fixos de irrigação. No caso dos sistemas autopropelidos, ainda falta uma análise mais completa dos efeitos das diferentes combinações de formas geométricas dos perfis e ângulos de giro do aspersor sobre os critérios de escolha do espaçamento entre carreadores. BITTINGER \& LONGENBAUGTH (1962), em um estudo limitado a aspersores operando com 
giro de $360^{\circ}$, concluíram que, para o caso de perfis triangulares e elípticos, os melhores espaçamentos entre carreadores correspondem a, respectivamente, $50 \%$ e $70 \%$ do diâmetro molhado. KELLER \& BLIESNER (1990), por considerarem que os canhões apresentam um perfil radial quase retangular, recomendam, para a condição de ventos calmos, um espaçamento entre carreadores próximo de $80 \%$ do diâmetro molhado e ângulo de giro na faixa entre 210 e $240^{\circ}$.

De modo a efetuar uma análise mais completa dos efeitos das diferentes formas geométricas do perfil radial de distribuição de água e do ângulo de giro de aspersores operando em sistemas autopropelidos de irrigação, este trabalho objetivou: i) analisar a influência da forma geométrica (triangular, elíptica e retangular) do perfil radial e do ângulo de giro sob a distribuição de água de aspersores em movimento linear, constante e uniforme, e ii) apresentar e analisar os valores de coeficiente de uniformidade de aplicação de água sob diferentes combinações de espaçamento entre carreadores e ângulo de giro do aspersor para as três formas geométricas típicas (triangular, elíptica e retangular) do perfil radial.

\section{MATERIAL E MÉTODOS}

O trabalho foi conduzido a partir de simulações digitais com auxílio de um aplicativo computacional, elaborado em Visual Basic da planilha eletrônica Excel, seguindo o modelo descrito por PRADO (2004). Este programa, conforme apresentado no fluxograma da Figura 1, a partir dos dados de vazão, raio de alcance, perfil radial de distribuição de água, velocidade de deslocamento no carreador e ângulo de giro do aspersor, simula a distribuição espacial da lâmina aplicada em um plano perpendicular à trajetória linear de deslocamento de um aspersor isolado, na condição de ausência de ventos, e calcula a uniformidade de aplicação de água do sistema de irrigação.

Levando em conta que a razão entre a vazão e o raio de alcance de um canhão hidráulico ensaiado por PRADO \& COLOMBO (2007) abrange valores entre 0,5 e 1,35, no desenvolvimento das simulações, foram considerados valores de vazão e raio de alcance seguindo uma relação de $1: 1$, ou seja, os valores de vazão $\left(30 ; 35 ; 40 ; 45 ; 50 ; 55\right.$, e $\left.60 \mathrm{~m}^{3} \mathrm{~h}^{-1}\right)$ foram numericamente iguais aos respectivos valores de raio de alcance, expresso em metros. Para as três formas geométricas (triangular, elíptica e retangular) do perfil radial de distribuição de água consideradas (Figura 2), os valores de intensidade de precipitação, em função da distância radial ao aspersor, para o aspersor operando com um ângulo de giro de $360^{\circ}$, foram calculados por:

- Perfil Triangular:

$$
i(r)=\left\{\begin{array}{l}
\text { Para }: 0 \leq r \leq R \rightarrow i(r)=\frac{3000 Q(R-r)}{\pi R^{3}} \\
\text { Para }: r>R \rightarrow i(r)=0
\end{array}\right.
$$

- Perfil Elíptico:

$$
\mathrm{i}(\mathrm{r})=\left\{\begin{array}{l}
\text { Para }: 0 \leq \mathrm{r} \leq \mathrm{R} \rightarrow \mathrm{i}(\mathrm{r})=\frac{1500 \mathrm{Q} \sqrt{\mathrm{R}^{2}-\mathrm{r}^{2}}}{\pi \mathrm{R}^{3}} \\
\text { Para }: \mathrm{r}>\mathrm{R} \rightarrow \mathrm{i}(\mathrm{r})=0
\end{array}\right.
$$

- Perfil Retangular:

$$
\mathrm{i}(\mathrm{r})=\left\{\begin{array}{l}
\text { Para }: 0 \leq \mathrm{r} \leq \mathrm{R} \rightarrow \mathrm{i}(\mathrm{r})=\frac{1000 \mathrm{Q}}{\pi \mathrm{R}^{2}} \\
\text { Para }: \mathrm{r}>\mathrm{R} \rightarrow \mathrm{i}(\mathrm{r})=0
\end{array}\right.
$$

em que,

i(r) - intensidade de precipitação do aspersor em função da distância radial, $\mathrm{mm} \mathrm{h}^{-1}$;

$\mathrm{r}$ - distância radial ao aspersor, m;

$\mathrm{Q}$ - vazão do aspersor, $\mathrm{m}^{3} \mathrm{~h}^{-1}$;

$\mathrm{r}$ - raio de alcance do aspersor, $\mathrm{m}$. 


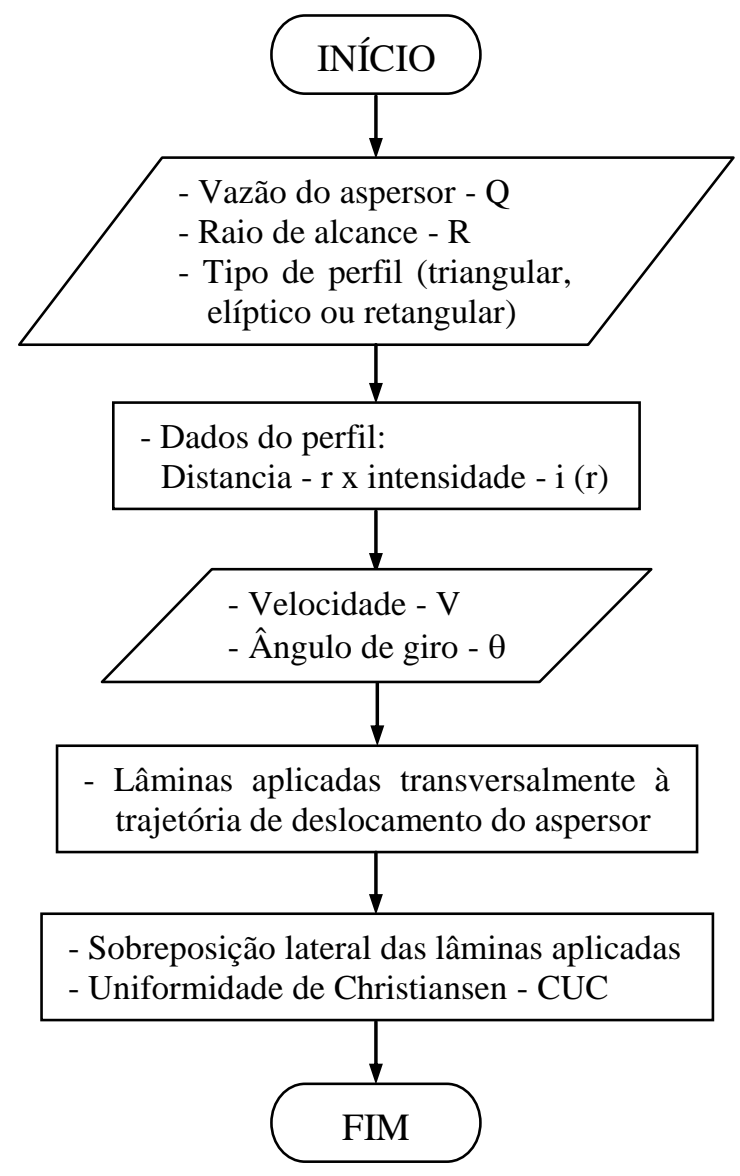

FIGURA 1. Fluxograma do programa para analisar a distribuição de água de equipamentos autopropelidos de irrigação com aspersores apresentando perfil radial triangular, elíptico ou retangular. Flowchart of the software to analyze the water distribution of traveler irrigation machines with sprinklers presenting triangular, elliptical or rectangular radial profile.

$\mathrm{O}$ aumento da intensidade de precipitação no interior do setor molhado do aspersor, decorrente do uso dos demais ângulos de giro $\left(\theta=180 ; 210 ; 240 ; 270 ; 300\right.$, e $\left.330^{\circ}\right)$, foi obtido multiplicando-se os valores de i(r) por uma constante de valor igual a 360/0. Em todas as simulações, foi considerado o mesmo valor de velocidade linear de deslocamento do aspersor $\left(50 \mathrm{~m} \mathrm{~h}^{-1}\right)$.


Distância ao aspersor - $\mathbf{r}(\mathbf{m})$

FIGURA 2. Perfis radiais de distribuição de água (triangular, elíptico e retangular) de aspersores com vazão de $50 \mathrm{~m}^{3} \mathrm{~h}^{-1}$ e raio de alcance de $50 \mathrm{~m}$. Radial water distribution profiles (triagular, elliptical and rectangular) of sprinklers with dischange of $50 \mathrm{~m}^{3} \mathrm{~h}^{-1}$ and radius of throw of $50 \mathrm{~m}$. 
A uniformidade de aplicação de água, em diferentes espaçamentos entre carreadores, compreendidos entre 0 a $100 \%$ do diâmetro molhado, foi obtida pela sobreposição lateral dos valores simulados de lâmina de água aplicada nos perfis transversais, tomados no centro da faixa irrigada pelo aspersor isolado. A uniformidade de aplicação de água, dos valores sobrepostos, foi quantificada por meio do coeficiente de uniformidade de Christiansen, usualmente expresso como CUC (CHRISTIANSEN, 1942), dado pela expressão:

$$
\mathrm{CUC}=\left(1-\frac{\sum_{\mathrm{j}=1}^{\mathrm{n}}\left|\mathrm{Is}_{\mathrm{j}}-\overline{\mathrm{Is}}\right|}{\mathrm{n} \overline{\mathrm{Is}}}\right) 100
$$

em que,

CUC - coeficiente de uniformidade de Christiansen, \%;

$\mathrm{Is}_{\mathrm{j}}$ - lâmina sobreposta observada em um ponto de amostragem de índice j, mm;

Is - lâmina sobreposta média, mm, e

n - número de pontos de amostragem.

As simulações da uniformidade de aplicação de água, em diferentes combinações de ângulo de giro do aspersor e espaçamento entre carreadores, foram agrupadas em três gráficos de superfície distintos, cada um dos quais representando uma mesma forma geométrica do perfil radial de distribuição de água do aspersor (triangular, elíptico ou retangular).

\section{RESULTADOS E DISCUSSÃO}

Na Figura 3, apresenta-se a distribuição da lâmina de água em cortes transversais tomados no centro da faixa irrigada por um aspersor isolado em deslocamento linear. Para o caso do perfil radial retangular, os resultados apresentados confirmam três afirmativas de KELLER \& BLIESNER (1990), referentes ao do ângulo de giro do aspersor: (i) o perfil de $360^{\circ}$ é exatamente igual ao de $180^{\circ}$; (ii) os valores máximos de lâmina do perfil perpendicular decrescem quando o ângulo de giro aumenta de $180^{\circ}$ para de $240^{\circ}$, e voltam a crescer quando o ângulo de giro passa de $240^{\circ}$ para $360^{\circ}$; (iii) perfis mais uniformes são obtidos com ângulos de giro compreendidos entre $210^{\circ}$ e $240^{\circ}$.

Como pode ser observado na Figura 3, as três considerações efetuadas por KELLER \& BLIESNER (1990), quanto às lâminas de água aplicadas transversalmente ao sentido de deslocamento do aspersor, também podem ser aplicadas para os equipamentos autopropelidos dotados de aspersores que apresentam perfil radial de distribuição de água com formato geométrico triangular e elíptico. Na Figura 4, são apresentados, para as três formas geométricas do perfil radial de distribuição de água analisadas, os valores de uniformidade de aplicação de água em função de diferentes combinações de ângulo de giro do aspersor e espaçamento entre carreadores. Nesta figura, os espaçamentos entre carreadores foram quantificados em termos de porcentagem do diâmetro molhado.

Observa-se, na Figura 4, que, para os perfis de formato triangular e elíptico, operando com ângulo de giro de $360^{\circ}$, os resultados mostrados confirmam a superioridade, observada por BITTINGER \& LONGENBAUGTH (1962), dos espaçamentos de, respectivamente, 50 e $70 \%$ do diâmetro molhado. No entanto, para ângulos de giro compreendidos entre $180^{\circ}$ e $360^{\circ}$, os valores indicam que os melhores valores de coeficiente de uniformidade de aplicação de água, para esses dois perfis, não são observados nestes mesmos espaçamentos. Tanto no perfil triangular como no perfil elíptico, nos mesmos espaçamentos recomendados por BITTINGER \& LONGENBAUGTH (1962), a redução no valor do coeficiente de uniformidade de aplicação de água é acentuada, quando o ângulo de giro do aspersor se aproxima de $270^{\circ}$. No caso do perfil retangular, observa-se também a existência de uma região de reduzida uniformidade com ângulos de giro entre $230^{\circ} \mathrm{e}$ $330^{\circ}$. 

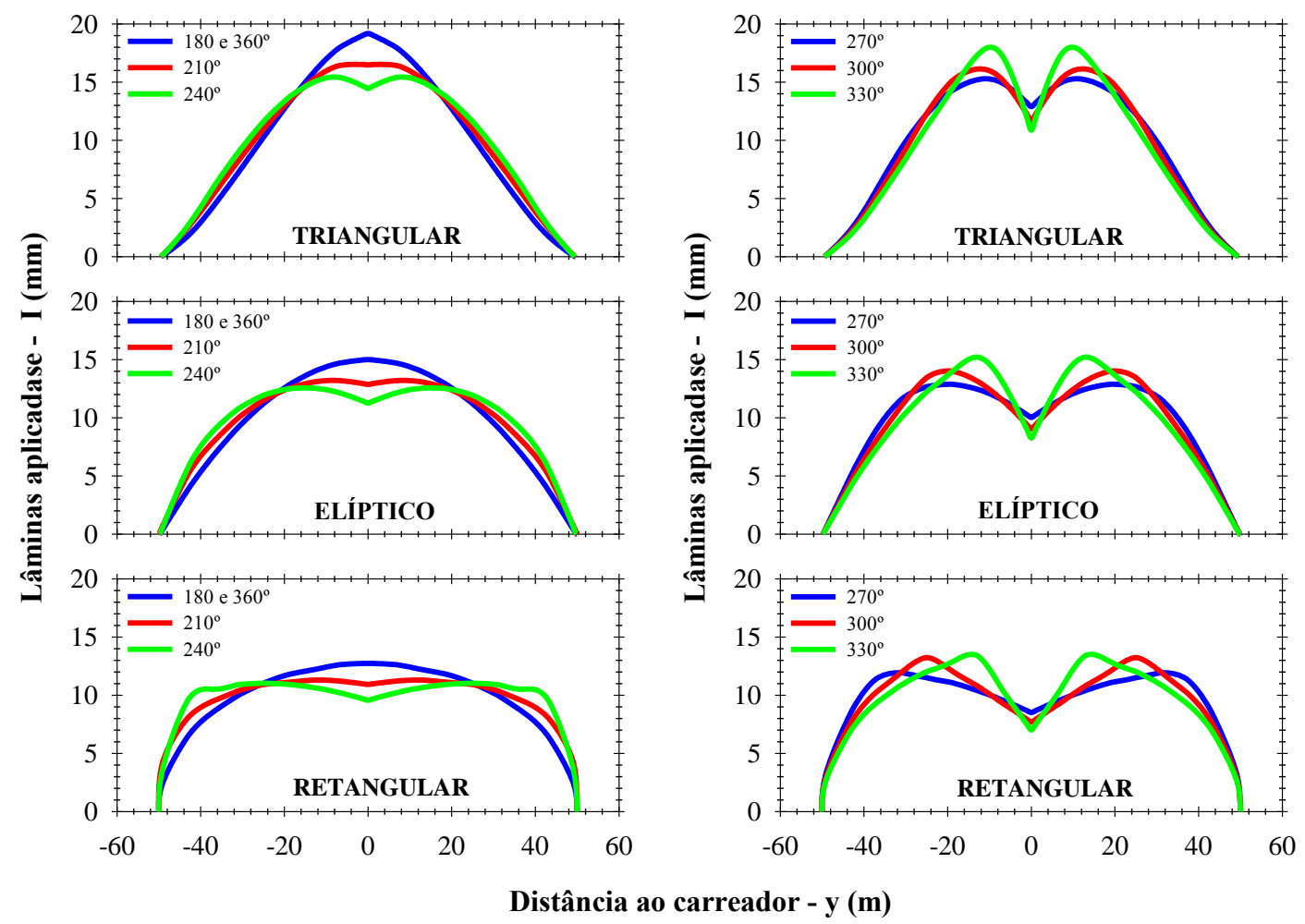

FIGURA 3. Simulações da lâmina aplicada em um plano perpendicular à trajetória linear de deslocamento de um aspersor com velocidade de $50 \mathrm{~m} \mathrm{~h}^{-1}$. Simulation of applied depths along a transect perpendicular to the travel direction of a sprinkler with a $50 \mathrm{~m} \mathrm{~h}^{-1}$ speed.

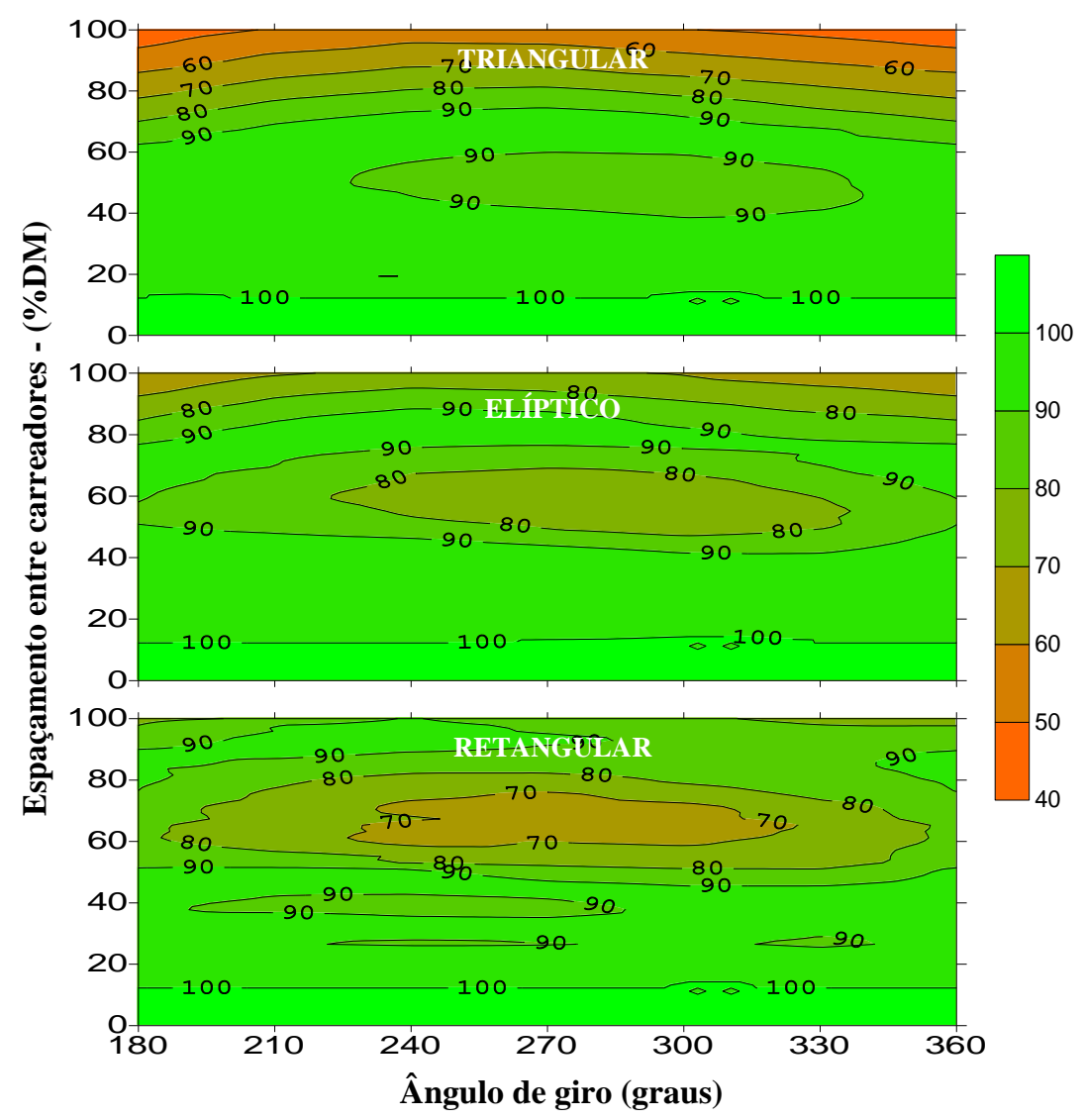

FIGURA 4. Coeficiente de uniformidade de Christiansen (CUC) para os três perfis radiais de distribuição de água (triangular, elíptico e retangular). Christiansen uniformity coefficient (CUC) for the three radial water distribution profiles (triangular, elliptical and rectangular). 
A operação de sistemas autopropelidos de irrigação com aspersores trabalhando com o ângulo de giro igual a $270^{\circ}$, que é uma prática comumente empregada por irrigantes e recomendada por projetistas de sistemas de irrigação (ROCHA et al., 2005), deve se revista, de modo a buscar uma otimização da aplicação de água desses equipamentos. Conforme evidenciado na Figura 4, independentemente do tipo de perfil radial de distribuição de água, ângulos de giro próximos de $270^{\circ}$ proporcionam baixos valores de coeficiente de uniformidade de aplicação de água.

Para a otimização das condições operacionais de sistemas autopropelidos de irrigação, visando à melhoria na distribuição de água desses equipamentos, os melhores valores de coeficiente de uniformidade de aplicação de água, de acordo com a Figura 4, são conseguidos com: $i$ ) ângulos de giro entre $180^{\circ}$ e $230^{\circ}$; ii) espaçamentos de carreadores menores que $45 \%$ e entre $55 \%$ a $70 \%$ do diâmetro molhado, para o perfil triangular; iii) espaçamentos de carreadores menores que $50 \%$ e entre $70 \%$ a $90 \%$ do diâmetro molhado, para os perfis elíptico e uniforme.

PRADO \& COLOMBO (2007), ao analisar a distribuição de água do canhão hidráulico PLONA-RL250, encontraram adequados valores de coeficiente de uniformidade de aplicação de água para espaçamentos entre carreadores e ângulos de giro do aspersor próximos aos observados para os perfis teóricos elíptico e retangular. Esta similaridade encontrada no trabalho destes autores é devida ao fato de o aspersor apresentar, para valores adequados de pressão de serviço, perfis radiais com formas geométricas que tendem para o perfil retangular. Desta forma, na ausência de informações sobre o perfil estacionário de distribuição de água de canhões hidráulicos, é mais conveniente optar pela forma geométrica elíptica ou retangular do perfil radial de distribuição de água, ao invés da forma geométrica triangular adotada por ROLIM \& PEREIRA (2005).

\section{CONCLUSÕES}

Nas três formas geométricas empregadas para o perfil radial de distribuição de água, especialmente em condições de espaçamento entre carreadores maiores que $45 \%$ do diâmetro molhado pelo aspersor, existe influência do ângulo de giro sobre os valores de coeficiente de uniformidade de aplicação de água. Desta forma, recomenda-se identificar, para os diversos modelos de aspersores, as combinações de ângulo de giro e espaçamento entre carreadores que resultam em melhor uniformidade de aplicação de água.

\section{REFERÊNCIAS}

ÁLVAREZ, J.F.O.; MARTIN-BENITO, J.M.T.; VALERO, J.A.J.; PÉREZ, P.C. Uniformity distribution and its economic effect on irrigation management in semiarid zones. Journal of Irrigation and Drainage Engineering, New York, v.130, n.4, p.257-268, 2004.

BITTINGER, M.W.; LONGENBAUGH, R.A. Theoretical distribution of water from a moving irrigation sprinkler. Transactions of the American Society of Agricultural Engineers, St. Joseph, v.5, n.1, p.26-30, 1962.

CÉSAR, J.H.F.; SILVA, A.M.; ROCHA, F.A.; MELLO, C.R. Avaliação técnica de um aspersor canhão do tipo turbina. Ciência Agrotécnica, Lavras, v.28, n.4, p.932-941, 2004.

CHRISTIANSEN, J.E. Irrigation by Sprinkling. Berkeley: California Agricultural Station, 1942. 124 p. (Bulletin, 670).

COLLIER, R.L.; ROCHESTER, E.W. Water application uniformity of hose towed traveler irrigators. Transactions of the American Society of Agricultural Engineers, St. Joseph, v.23, n.5, p.1.135-1.138, 1980.

COLOMBO, A. Simulação do desempenho de um equipamento autopropelido de irrigação. 1991. 120 f. (Dissertação) - Escola Superior de Agricultura "Luiz de Queiroz", Universidade de São Paulo, Piracicaba, 1991. 
FARIA, L.C.; COLOMBO, A.; OLIVEIRA, H.F.E.; PRADO, G. Simulação da uniformidade de irrigação de sistemas convencionais de aspersão operando sob diferentes condições de vento. Engenharia Agrícola, Jaboticabal, v.29, n.1, p.19-27, 2009.

KELLER, J.; BLIESNER, R.D. Sprinkle and trickle irrigation. New York: Van Nostrand Reinhold, $1990.652 \mathrm{p}$.

NEWELL, G.F.; FOLEY, J.; SMITH, R. TRAVGUN-Travelling Gun Simulation model user's manual. Version 1.1. Toowooba: QLD, 2003.91 p.

PAZ, V.P.S.; FRIZZONE, J.A.; BOTREL, T.A.; FOLEGATTI, M.V. Otimização do uso da água em sistema de irrigação por aspersão. Revista Brasileira de Engenharia Agrícola e Ambiental, Campina Grande, v.6, n.3, p.404-408, 2002.

PRADO, G. Aplicativo computacional para simulação da distribuição de água pelo aspersor PLONA-RL300 em sistemas autopropelidos de irrigação. 2004. 86 p. Dissertação (Mestrado em Irrigação e Drenagem) - Universidade. Federal de Lavras, Lavras, 2004.

PRADO, G. Modelagem da aplicação de água com canhões hidráulicos sob diferentes condições de vento. 2008. 121 p. Tese (Doutorado em Engenharia de Água e Solo) - Universidade Federal de Lavras, Lavras, 2008.

PRADO, G.; COLOMBO, A. Análise da uniformidade de aplicação de água pelo aspersor PLONARL250 em sistemas autopropelidos de irrigação. Irriga, Botucatu, v.12, n.2, p.249-262, 2007.

PRADO, G.; COLOMBO, A. Aplicativo computacional para simulação da distribuição espacial de água aplicada por canhões hidráulicos em sistemas autopropelidos de irrigação. In: CONGRESSO NACIONAL DE IRRIGAÇÃO E DRENAGEM, 16., 2006, Goiânia. Anais... Brasília: ABID, 2006.

PRADO, G.; COLOMBO, A. Caracterização técnica do aspersor PLONA-RL300. Irriga, Botucatu, v.10, n.1, p.53-63, 2005.

PRADO, G.; COLOMBO, A.; BIAGIONI, P. F. Análise da uniformidade de aplicação de água pelo aspersor PLONA-RL400 em sistemas autopropelidos de irrigação. Engenharia Agrícola, Jaboticabal, v.27, n.2, p.346-353, 2007.

ROCHA, F.A.; PEREIRA, G.M.; ROCHA, F.S.; SILVA, J.O. Análise da uniformidade de distribuição de água de um equipamento autopropelido. Irriga, Botucatu, v.10, n.1, p.96-106, 2005.

ROLIM, J.; PEREIRA, L.S. Design and evaluation of traveling-gun systems: the simulation model TRAVGUN. In: EFITA/WCCA Joint Congress on IT in Agriculture, 2005, Vila Real. Proceedings... Vila Real: EFITA/WCCA, 2005. p.166-171.

SATURNINO, H.M. Cerrados podem tornar-se um imenso canavial. Revista Item, Brasília, n.69/70, p.73-75, 2006.

SHULL, H.; DYLLA, A.S. Traveling gun application uniformity in high winds. Transactions of the American Society of Agricultural Engineers, St. Joseph, v.19, n.2, p.254-258, 1976.

SMITH, R.; GILLIES, M. H.; NEWELL, G.; FOLEY, J. P. A decision support model for travelling gun irrigation machines. Biosystems Engineering, Amsterdam, v.100, n.1, p.126-136, 2008. 